\title{
Response preparation and code overlap in dual tasks
}

\author{
IRING KOCH and WOLFGANG PRINZ \\ Max Planck Institute for Human Cognitive and Brain Sciences, Munich, Germany
}

\begin{abstract}
In a dual-task paradigm, a visual-encoding task with a deferred verbal report of a moving target was combined with a speeded task, in which participants prepared a precued leftward or rightward keypress response that was withheld until an auditory go signal. We manipulated the interval between the response cue and the target for the visual-encoding task, the interval between this target and the go signal, and spatial cross-task compatibility between the direction of the target movement in the visual task and the speeded manual response. The results of two experiments suggest that visual encoding interferes with response preparation and with the initiation of the prepared manual response at a short target-go interval. Also, responses were faster in compatible than in incompatible trials, indicating a cross-task compatibility effect. Experiment 2 reversed this compatibility effect by instruction, suggesting that the compatibility effect is based on response-response overlap. In both experiments, response preparation impaired accuracy in the visual task. Taken together, these results suggest that response processes and visual encoding share common codes and processes.
\end{abstract}

Dual-task experiments represent a major method for studying the functional characteristics of the cognitive system (e.g., Meyer \& Kieras, 1999). For instance, when two stimuli are sequentially presented and participants respond as quickly as possible to the stimuli in order of appearance, the second response is slowed down when the interstimulus interval is shortened (see Pashler, 1994, for a review). More recently, it has been shown that such dual-task interference is not restricted to the selection of two speeded responses but also extends to memory retrieval (e.g., Carrier \& Pashler, 1995) and encoding (e.g., Jolicœur \& Dell'Acqua, 1998, 1999), suggesting that the dual-task limitation (i.e., the cognitive bottleneck) is based on a rather broad memory process interference (see, e.g., Arnell \& Duncan, 2002; Jolicœur, Tombu, Oriet, \& Stevanovski, 2002; Logan \& Gordon, 2001; Pashler, 2000).

However, memory process interference may not be the only source of dual-task interference. In addition, there can be content-based interference. Such content-based interference can arise when two tasks draw on similar or identical representations. For instance, dual-task interactions can be based on dimensional overlap (see Kornblum, Hasbroucq, \& Osman, 1990) that arises from a similarity between codes (e.g., when two codes both refer to the spa-

We thank Robert D. Gordon, Pierre Jolicœur, Chris Oriet, Biljana Stevanovski, Michael Tombu, and an anonymous reviewer for helpful comments, as well as Marc Grosjean and Stefanie Schuch for helpful discussions of an earlier version of this article. Correspondence concerning this article should be addressed to I. Koch, Max Planck Institute for Human Cognitive and Brain Sciences, Department of Psychology, Amalienstrasse 33, D-80799 Munich, Germany (e-mail: iring.koch@ cbs.mpg.de). tial dimension). Dimensional overlap can produce crosstask compatibility (Koch \& Prinz, 2002) in dual tasks when the stimulus or response codes in one task are compatible with the stimulus or response codes in the other task.

The present study had two aims. One aim was to further investigate dual-task process interference. The other aim was to explore the representational basis of cross-task compatibility. In the following, we will describe our experimental paradigm.

\section{The Response-Cuing Dual-Task Paradigm}

In the present study, we combined a visual-encoding task, requiring a deferred verbal report, with a speeded manual reaction time (RT) task for which the response was precued (Koch \& Prinz, 2002). In the RT task, first a visual cue (a red or blue $X$ ) was presented in order to indicate a leftward or rightward keypress response, which, however, was to be withheld until an auditory go signal. In the visual-encoding task, the target stimulus was a briefly presented small dot that moved from screen center to the left or right and was followed by a mask. The participant verbally reported the direction of the movement. However, the two tasks were interleaved, so that the speeded response to the visual cue occurred during presentation of the mask and the unspeeded verbal response occurred at the end of the trial. Figure 1 presents the ordering of events within a trial.

Two intervals can be manipulated in this nested dual-task paradigm. The first interval is the cue-target interval (CTI), which is the time for response preparation undisturbed by the presentation of the visual target. The second interval is the target-go interval (TGI), which represents the time for encoding and consolidating the visual target prior to the auditory go signal. 


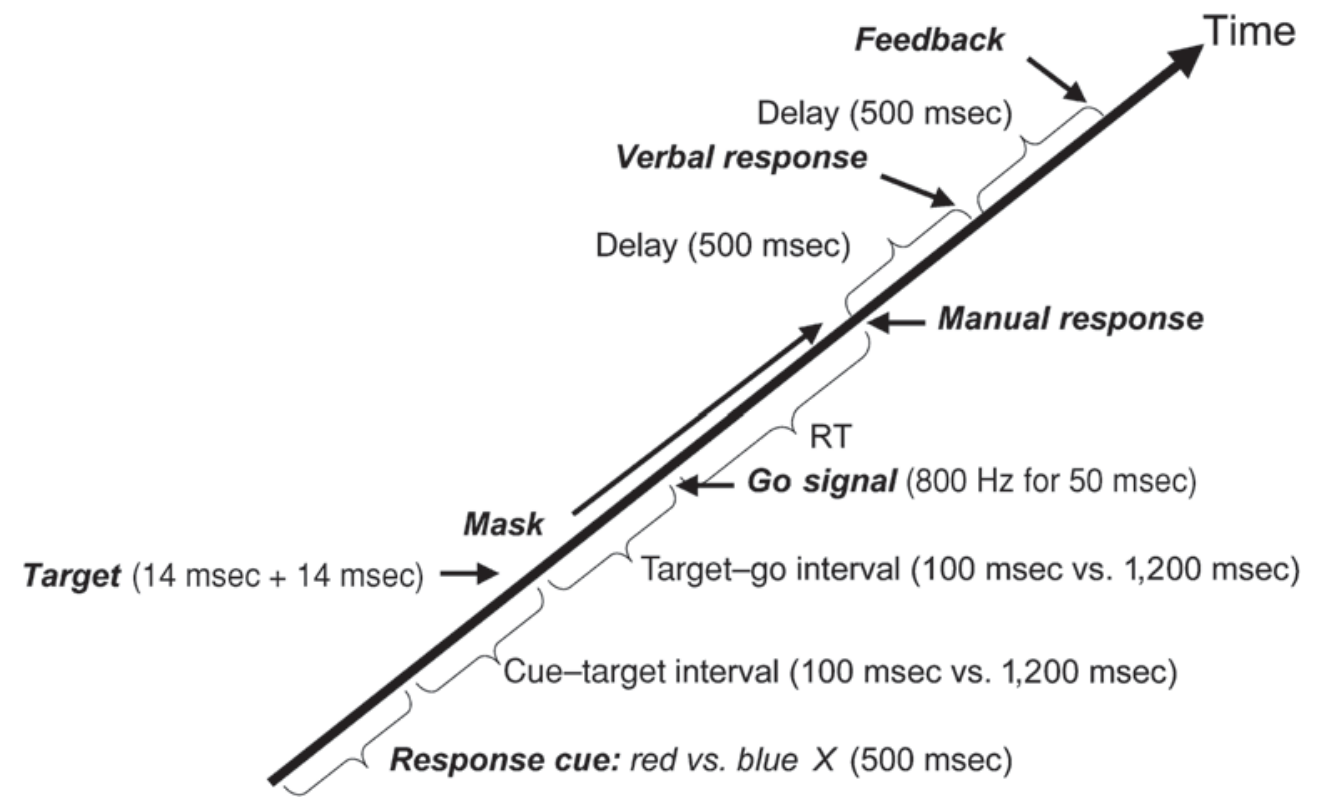

Figure 1. Schema of the experimental procedure in the nested dual-task paradigm. A color precue indicated whether a speeded manual response to the go signal would be a left or a right keypress. A briefly presented visual target that moved to the left or the right required an unspeeded verbal response.

\section{Response Preparation and Dual-Task Process Interference}

In a previous study, we found that encoding in the visual task postpones the subsequent initiation of the precued response in the other task (Koch \& Prinz, 2002). This finding extended earlier findings of encoding-based dual-task interference with speeded choice reactions to precued responses (e.g., Jolicœur \& Dell'Acqua, 1998; see Jolicœur et al., 2002, for a review). That is, visual encoding appears to produce a kind of processing bottleneck with respect to response initiation. If that is the case, one could hypothesize that any potentially beneficial effects of response preparation would be offset by the required intervening visual-encoding process. We tested this hypothesis in the present study.

The role of cue-based response preparation was not directly examined in our earlier study (Koch \& Prinz, 2002). For instance, in Experiment 1 of that study, we manipulated the TGI, while keeping the total interval between the response cue and the go signal constant. As a consequence, the CTI and the TGI varied inversely (e.g., $1,200 / 100 \mathrm{msec}$ vs. $100 / 1,200 \mathrm{msec}$ ). To isolate the interfering effect of visual encoding on response initiation in the other task, the CTI was kept constant in the third experiment, and only TGI was manipulated. Addressing the role of cue-based response preparation requires manipulating the CTI and the TGI independently.

In the present study, we used this independent manipulation to examine the effects of cue-based response preparation on process interference. If visual encoding indeed creates some kind of processing bottleneck with respect to the initiation of a prepared response, one should predict that RT to the go signal will not be affected by CTI duration, but only by TGI duration, because response preparation will be disrupted until the visual-encoding process is completed. Consequently, with a short TGI, the visualencoding process will delay the initiation of the cued responses, whereas a long TGI will allow completion of the visual-encoding process, so that, to some extent, response preparation can proceed without interference prior to the go signal (see Jolicœur \& Dell'Acqua, 1998; Koch \& Prinz, 2002).

To pinpoint the hypothesized disruptive role of visual encoding for the response preparation process, we compared dual-task performance with that in a single-task control condition in which no encoding of the visual target was necessary (no-report condition). If visual encoding disrupts response preparation, preparation benefits should be much larger in the no-report condition than in the report condition. This hypothesized interaction between CTI and the report/no-report variable should be particularly pronounced at the short TGI-that is, when there is not much time for response preparation after the onset of the visual target.

In addition, we also explored interactions of response preparation with visual encoding. Specifically, we explored whether response preparation speeds up the cued response at the cost of impairing the quality of visual encoding. Finding an effect of response preparation (i.e., the CTI) on accuracy in the visual task would support previously reported findings that response selection in choice RT tasks can impair performance in a subsequent visual task (see, e.g., De Jong \& Sweet, 1994; Jolicœur, 1999; Müsseler \& Wühr, 2002). It would also extend these find- 
ings, because it would imply that not only response selection, but also preparation of an already selected response produces interference with a visual task.

\section{Code Overlap in Dual Tasks}

In our earlier study (Koch \& Prinz, 2002), we observed that RT was shorter when the movement directions in both tasks were the same rather than different, indicating a cross-task compatibility effect. Note that crosstask compatibility effects are not a demonstration of the ubiquitous spatial stimulus-response (S-R) compatibility effect (see, e.g., Hommel \& Prinz, 1997), because the latter is confined to S-R code overlap within a task. Cross-task compatibility is a different phenomenon, because it refers to compatibility between tasks (see Lien \& Proctor, 2002; Logan \& Gordon, 2001). Importantly, this cross-task compatibility effect also differs from those dual-task code overlap effects previously reported in the literature. Whereas the present cross-task compatibility effect is based on dual-task priming from a nonspeeded visual-encoding task to a speeded, precued response task, previous effects pertained either to an effect between two speeded choice RT tasks (e.g., Hommel, 1998; Lien \& Proctor, 2000; Logan \& Gordon, 2001; Schuch \& Koch, 2004) or to an effect on accuracy in a nonspeeded visual task (e.g., Müsseler \& Hommel, 1997; Stevanovski, Oriet, \& Jolicœur, 2003).

The existence of dual-task compatibility effects in general suggests a substantial degree of cross-task interactions in dual-task performance (see Lien \& Proctor, 2002, for a review). Such effects are important theoretically because they emphasize the interactions of task performance on the level of cognitive codes (i.e., code overlap), whereas bottleneck notions emphasize nonoverlapping processes in dual-task performance (e.g., Pashler, 1993, 1994, for a review). It is thus a theoretically important issue to elucidate the representational basis of the cross-task compatibility effect identified in Koch and Prinz (2002). This effect could be based either on dimensional overlap between the code referring to the visual target and that of the prepared response (S-R) or on the overlap between the code representing the (deferred) response to the visual target and that of the prepared response (R-R).

If the cross-task compatibility effect is based on R-R overlap, this would be consistent principally with the idea that participants immediately generate a response code for the visual target, although a speeded response is not required, and that it is this response code that then interacts with the other response code. A cross-task compatibility effect based on R-R overlap would be consistent with a liberalized version of a response selection bottleneck account (e.g., Pashler, 2000) that allows covert (i.e., deferred) responses to have effects on subsequent response processes that are similar to those of overt responses.

We disentangled the two possible code overlap relations (i.e., S-R vs. R-R) by manipulating the encoding instruction for the visual task. Specifically, in Experiment 1 we asked participants to report the direction of the stimulus movement, whereas in Experiment 2 we asked them to report the "origin" of the movement, so that physically identical stimuli gave rise to opposite responses in the two experiments. If we find that the cross-task compatibility effect is reversed as a function of encoding instruction, this would suggest that the compatibility effect is due to $\mathrm{R}-\mathrm{R}$ overlap, rather than to $\mathrm{S}-\mathrm{R}$ overlap, for which the instruction manipulation should have no effect.

\section{Overview of Experiments}

In two experiments, we examined dual-task interactions of response preparation and visual encoding. The aim of Experiment 1 was to study the role of response preparation for process interference and also to manipulate cross-task compatibility. The aim of Experiment 2 was to disentangle the code overlap relations underlying cross-task compatibility.

\section{EXPERIMENT 1}

\section{Method}

Participants. Eighteen participants ( 15 of them female, 3 male), $20-30$ years of age, took part for $€ 7.50$ (about $\$ 7.50$ ).

Apparatus and Stimuli. The experiment was controlled by a PC interfaced to a 15-in. color screen. Stimulus presentation was synchronized with the vertical retraces of a $70.1-\mathrm{Hz}$ monitor. The response cue for the RT task was a red or a blue $X$ on a light-gray background, indicating a finger movement to either a left or a right key, respectively, counterbalanced over participants. The $X$ was presented at screen center and was approximately $1.5 \mathrm{~cm}$ high. The auditory go signal for the precued manual reaction was an $800-\mathrm{Hz}$ tone presented for $50 \mathrm{msec}$. It was emitted by the internal speaker of the PC, placed in front of the participants. The manual response to the go signal was given on an external keyboard with three response keys (1.8-cm width, $3.3 \mathrm{~cm}$ apart). Responses were executed by moving the dominant index finger from the center (home) key to one of the two side keys. The stimulus for the visual-encoding task was a black dot $(0.2 \mathrm{~cm})$ displayed for $14 \mathrm{msec}$ at screen center and then for another $14 \mathrm{msec}$ at a position $0.2 \mathrm{~cm}$ to the left or right, creating an impression of apparent motion. The dot was immediately followed by a mask $(5.6 \times 9.7 \mathrm{~cm})$ in which pixels were set at random. The participants' nonspeeded verbal report of the target movement direction at the end of the trial was entered by the experimenter.

Procedure. The participants read task instructions on the screen but were also verbally instructed. They were told to perform two tasks. For the RT task, a colored $X$ (i.e., the response cue) would appear at screen center, indicating that the participants should plan a leftward or rightward keypress response (i.e., a finger movement from the home key to the right or left key), depending on stimulus color. However, the participants were told to withhold the planned response until a go signal beeped. They were then informed that, after offset of the response precue, a moving target for the visual task would be presented briefly at screen center before the onset of the go signal and that they had to report the target movement direction after executing the speeded response. In some trials, the instruction on the screen also informed the participants explicitly that they could ignore the target on this specific trial and simply respond as quickly as possible to the go signal (no report). The initiation of each trial was self-paced, so that the participants had as much time as they needed to process this information before they started the trial.

The participants first ran through a practice block of 32 trials, followed by six experimental blocks with 64 trials each. Between blocks, a break was offered. Each trial started with a message indicating whether direction of the movement of the target needed to be reported (report vs. no report). It also reminded the participants of the color-to-response-key mapping, and it reminded them that 
they had to press the home key to start the next trial, keeping this key pressed until onset of the go signal. Pressing the home key triggered (after a 500-msec delay) the presentation of the response cue for $500 \mathrm{msec}$. Then the cue disappeared, and after a CTI of either 100 or 1,200 msec, the visual target was presented for $28 \mathrm{msec}$ (i.e., $14 \mathrm{msec}+14 \mathrm{msec}$ ), followed by the mask. The mask remained on the screen until the manual response was made. The TGI was either 100 or $1,200 \mathrm{msec}$. Neither interval was predictable. Either releasing the home key too early or pressing the wrong key was scored as an error. Visual error feedback appeared for $500 \mathrm{msec}$ on the lower part of the screen. No-report trials ended then, but for report trials another $500 \mathrm{msec}$ elapsed before the next message appeared on the screen, asking whether the target had moved to the left or the right. The participants answered verbally, and the experimenter entered the response, producing accuracy feedback on the screen. The experiment continued only after the participants had initiated the next trial. The experiment took about $50 \mathrm{~min}$.

Design. CTI (100 vs. 1,200 msec), TGI (100 vs. 1,200 msec), report (report vs. no report), and cross-task compatibility (compatible vs. incompatible) were independently manipulated as withinsubjects variables. In a compatible trial, the movement directions in both tasks were the same, whereas the directions were different in an incompatible trial. In each of the six experimental blocks, 48 report trials and 16 no-report trials occurred, with conditions randomly ordered. In total, this resulted in 36 observations for each of the eight conditions for the report trials and 12 for the no-report trials. The dependent measures for the cued-response task were both accuracy and RT, defined as the time to complete the response by hitting the left or the right response key. For the visual-encoding task, we measured accuracy. All statistical analyses were conducted with an alpha of .05 .

\section{Results and Discussion}

Visual-encoding task. For data analysis, we discarded trials with an incorrect response in the RT task. Mean percentage correct in the visual task (see Table 1, left part) was submitted to an ANOVA with independent variables of CTI, TGI, and cross-task compatibility. (For clarity, we report the accuracy data as percentage correct in the text, but the reported $M S_{\mathrm{e}} \mathrm{s}$ are generally based on proportion correct.) The only significant effect was that of CTI $\left[F(1,17)=9.11, M S_{\mathrm{e}}=0.006694, p<.01\right]$, indicating that accuracy was higher for the short CTI than for the long CTI ( $90.4 \%$ vs. $86.2 \%$, respectively). All other effects were nonsignificant (all $F \mathrm{~s}<1.2, p \mathrm{~s}>.3$ ). That is, we found a decrease in visual perceptual accuracy with increasing response preparation time, suggesting that preparing a response affects visual encoding in another task. An alternative possibility, which we will discuss in the
General Discussion section, might be that the decrease in visual perceptual accuracy with a long CTI is also due to an unspecific alertness effect.

Reaction time task. We excluded trials with incorrect verbal report from the analysis of the RT task data. For this analysis, we also excluded incorrect responses. Finally, we discarded trials with RTs exceeding 4,000 msec as outliers (fewer than $1 \%$ of the correct trials). Table 2 shows mean RTs, and Table 3 shows percentages correct as functions of CTI, TGI, cross-task compatibility, and report.

The RT and accuracy data were submitted to separate ANOVAs with within-subjects variables of CTI, TGI, cross-task compatibility, and report. Because the data show some speed-accuracy trade-offs, we will report the results of the two ANOVAs together. First, we will report the results referring to the role of response preparation.

We predicted that visual encoding should be disruptive for response preparation with the short TGI, so that the expected CTI $\times$ TGI interaction should be much more pronounced in no-report trials than in report trials. In fact, in the RT data, CTI, TGI, and report entered into a significant three-way interaction $\left[F(1,17)=4.93, M S_{\mathrm{e}}=\right.$ $6,624, p<.05]$. Separate tests of the CTI $\times$ TGI interaction for each level of report showed that the CTI effect was larger with the short than with the long TGI in the no-report condition $[95$ vs. $-4 \mathrm{msec} ; F(1,17)=11.48$, $\left.M S_{\mathrm{e}}=7,711, p<.01\right]$, but not in the report condition $(27$ vs. $14 \mathrm{msec} ; F<1)$. This shows that the RT benefit of the long CTI when the TGI was short was restricted mostly to the no-report condition. This three-way interaction was not significant in the accuracy data $(F<1)$.

We also found significant main effects of CTI, TGI, and report, as well as two-way interactions between CTI and TGI and between TGI and report. However, the interpretation of these effects is complicated by speed-accuracy trade-offs that were not observed for the predicted threeway interaction described above. Specifically, RT was $33 \mathrm{msec}$ longer with the short CTI than with the long CTI $\left[F(1,17)=8.43, M S_{\mathrm{e}}=9,353, p<.05\right]$. However, accuracy was also $2.3 \%$ higher with the short CTI than with the long CTI $\left[F(1,17)=5.84, M S_{\mathrm{e}}=0.006638\right.$, $p<.05]$. Also, we found that RT was $281 \mathrm{msec}$ longer with the short TGI than with the long TGI $[F(1,17)=$ $\left.72.92, M S_{\mathrm{e}}=77,820, p<.01\right]$. But again, accuracy was

Table 1

Mean Percentages Correct for the Visual-Encoding Task in Experiments 1 and 2 as a Function of Target-Go Interval (TGI), Cue-Target Interval (CTI), and Cross-Task Compatibility

\begin{tabular}{|c|c|c|c|c|c|c|c|c|}
\hline \multirow[b]{3}{*}{ Condition } & \multicolumn{4}{|c|}{ Experiment 1} & \multicolumn{4}{|c|}{ Experiment 2} \\
\hline & \multicolumn{2}{|c|}{ 100-msec TGI } & \multicolumn{2}{|c|}{ 1,200-msec TGI } & \multicolumn{2}{|c|}{ 100-msec TGI } & \multicolumn{2}{|c|}{ 1,200-msec TGI } \\
\hline & $M$ & $S D$ & $M$ & $S D$ & $M$ & $S D$ & $M$ & $S D$ \\
\hline \multicolumn{9}{|l|}{$\mathrm{CTI}=100 \mathrm{msec}$} \\
\hline Incompatible & 89.5 & 9.4 & 89.4 & 12.4 & 92.0 & 9.7 & 89.3 & 14.3 \\
\hline Compatible & 91.0 & 12.1 & 91.5 & 8.0 & 91.7 & 9.8 & 91.1 & 10.7 \\
\hline \multicolumn{9}{|l|}{$\mathrm{CTI}=1,200 \mathrm{msec}$} \\
\hline Incompatible & 85.1 & 14.3 & 86.8 & 10.6 & 86.1 & 12.6 & 88.7 & 10.0 \\
\hline Compatible & 87.4 & 11.8 & 85.6 & 12.6 & 84.8 & 14.0 & 84.6 & 16.4 \\
\hline
\end{tabular}


Table 2

Reaction Times (in Milliseconds) in Experiment 1 as a Function of Report, Target-Go Interval (TGI), Cue-Target Interval (CTI), and Cross-Task Compatibility

\begin{tabular}{|c|c|c|c|c|c|c|c|c|}
\hline \multirow[b]{3}{*}{ Condition } & \multicolumn{4}{|c|}{ Report } & \multicolumn{4}{|c|}{ No Report } \\
\hline & \multicolumn{2}{|c|}{ 100-msec TGI } & \multicolumn{2}{|c|}{ 1,200-msec TGI } & \multicolumn{2}{|c|}{ 100-msec TGI } & \multicolumn{2}{|c|}{ 1,200-msec TGI } \\
\hline & $M$ & $S D$ & $M$ & $S D$ & $M$ & $S D$ & $M$ & $S D$ \\
\hline \multicolumn{9}{|l|}{$\mathrm{CTI}=100 \mathrm{msec}$} \\
\hline Incompatible & 1,063 & 365 & 647 & 289 & 829 & 329 & 603 & 177 \\
\hline Compatible & 1,020 & 353 & 650 & 239 & 799 & 292 & 575 & 197 \\
\hline \multicolumn{9}{|l|}{$\mathrm{CTI}=1,200 \mathrm{msec}$} \\
\hline Incompatible & 1,040 & 381 & 649 & 258 & 708 & 261 & 599 & 197 \\
\hline Compatible & 989 & 372 & 622 & 219 & 730 & 293 & 586 & 193 \\
\hline
\end{tabular}

$6.3 \%$ higher with the short TGI than with the long TGI $\left[F(1,17)=32.70, M S_{\mathrm{e}}=0.008605, p<.01\right]$. Finally, RT was $156 \mathrm{msec}$ longer in report trials than in no-report trials $\left[F(1,17)=30.92, M S_{\mathrm{e}}=56,870, p<.01\right]$, but performance was also $2.7 \%$ more accurate in report than in no-report trials $\left[F(1,17)=9.76, M S_{\mathrm{e}}=0.005404, p<\right.$ $.01]$. There were significant two-way interactions of CTI and TGI for RT $\left[F(1,17)=12.95, M S_{\mathrm{e}}=4,452, p<.01\right]$ and for accuracy $\left[F(1,17)=6.22, M S_{\mathrm{e}}=0.003437, p<\right.$ $.05]$. This interaction was due to the fact that prolonging the CTI led to a $62-\mathrm{msec}$ benefit in the short TGI, whereas this benefit was only $5 \mathrm{msec}$ at the long TGI. However, the effect for accuracy showed that the CTI effect was smaller with the short TGI than with the long TGI $(0.6 \%$ vs. $4.1 \%$ ). TGI and report interacted significantly as well, for both RT $\left[F(1,17)=72.23, M S_{\mathrm{e}}=11,003, p<.01\right]$ and accuracy $\left[F(1,17)=7.29, M S_{\mathrm{e}}=0.003381, p<\right.$ $.05]$. The effect of TGI was larger in report trials $(1,028$ vs. $642 \mathrm{msec}$, with the short and the long TGIs, respectively) than in no-report trials (766 vs. $591 \mathrm{msec}$, with the short and the long TGIs, respectively), but for accuracy the effect was smaller in report trials $(98.4 \%$ vs. $94.0 \%$ with the short and the long TGIs, respectively) than in no-report trials ( $97.6 \%$ vs. $89.5 \%$ with the short and the long TGIs, respectively). That is, the RT effects were associated with effects in the accuracy data that went in the opposite direction. Importantly, we note that the predicted three-way interaction of CTI, TGI, and report, which was significant for RT, was not significant in the accuracy data $(F<1)$, so that there was no clear speed-accuracy tradeoff here.
In sum, with respect to the effects of response preparation on RT, we observed that prolonging the CTI had its effects mainly in no-report trials with the short TGI. That is, even though response preparation for the RT task decreased perceptual accuracy (by 4.2\%) in the encoding task on report trials, it resulted only in a very modest decrease in RTs (roughly $20 \mathrm{msec}$ ). In no-report trials, however, response preparation during the CTI affected performance only in the short TGI condition, because lack of response preparation with a short CTI could be compensated for by preparation during the TGI when this was long enough.

Furthermore, in report trials, we observed a clear effect of visual encoding on RT in the cued-response task. With the short TGI, we found substantial response postponement, replicating previous findings of encoding-based dual-task interference (see Jolicœur et al., 2002; Koch \& Prinz, 2002). Thus, we found evidence that both tasks suffer from dual-task interference.

With respect to code-specific dual-task effects, we found a significant effect of cross-task compatibility $\left[F(1,17)=7.57, M S_{\mathrm{e}}=4,073, p<.05\right]$, reflecting the fact that RT was $21 \mathrm{msec}$ shorter in compatible than in incompatible trials. Corresponding to the RT effect, accuracy was slightly (but not significantly) higher in compatible trials than in incompatible trials. The RT effect was qualified by an unpredicted three-way interaction of cross-task compatibility, report, and CTI $[F(1,17)=$ 7.57, $M S_{\mathrm{e}}=1,668, p<.05$; see Figure 2]. This shows that the cross-task compatibility effect in report trials was smaller with the short CTI than with the long CTI (20 vs.

Table 3

Percentages Correct in Experiment 1 as a Function of Report, Target-Go Interval (TGI), Cue-Target Interval (CTI), and Cross-Task Compatibility

\begin{tabular}{|c|c|c|c|c|c|c|c|c|}
\hline \multirow[b]{3}{*}{ Condition } & \multicolumn{4}{|c|}{ Report } & \multicolumn{4}{|c|}{ No Report } \\
\hline & \multicolumn{2}{|c|}{ 100-msec TGI } & \multicolumn{2}{|c|}{ 1,200-msec TGI } & \multicolumn{2}{|c|}{ 100-msec TGI } & \multicolumn{2}{|c|}{ 1,200-msec TGI } \\
\hline & $M$ & $S D$ & $M$ & $S D$ & $M$ & $S D$ & $M$ & $S D$ \\
\hline \multicolumn{9}{|l|}{$\mathrm{CTI}=100 \mathrm{msec}$} \\
\hline Incompatible & 98.7 & 2.1 & 95.8 & 4.0 & 98.2 & 3.6 & 91.2 & 8.8 \\
\hline Compatible & 99.2 & 2.0 & 96.4 & 2.5 & 97.2 & 5.7 & 91.7 & 9.9 \\
\hline \multicolumn{9}{|l|}{$\mathrm{CTI}=1,200 \mathrm{msec}$} \\
\hline Incompatible & 97.7 & 2.6 & 92.0 & 6.1 & 96.8 & 6.5 & 87.5 & 13.5 \\
\hline Compatible & 98.2 & 2.9 & 91.9 & 6.2 & 98.2 & 3.6 & 87.5 & 13.2 \\
\hline
\end{tabular}




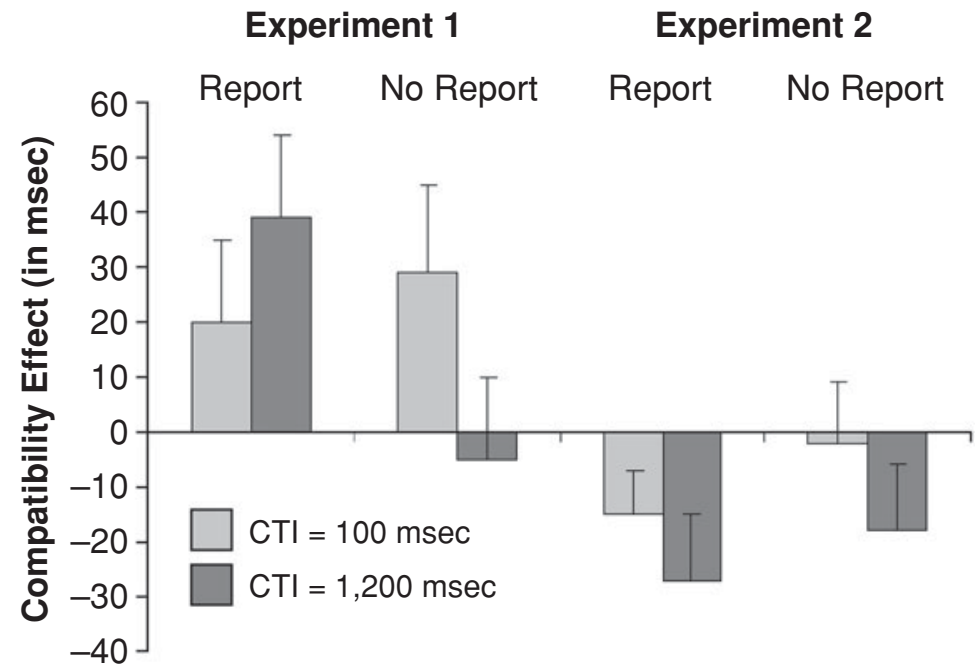

Figure 2. Cross-task compatibility effect (in milliseconds) in Experiments 1 and 2 as a function of report and cue-target interval (CTI). Error bars represent standard errors. The instructions for the visual-encoding task emphasized the endpoint of the target stimulus movement in Experiment 1 and the starting point in Experiment 2.

$39 \mathrm{msec})$, whereas this pattern was reversed in no-report trials ( 29 vs. $-5 \mathrm{msec})$. Put differently, response preparation with a long CTI served to effectively abolish the cross-task compatibility effect in no-report trials, whereas it even increased the size of the cross-task compatibility effect in report trials, in which response preparation was relatively ineffective at speeding up the cued response. However, when the CTI $\times$ cross-task compatibility interaction was tested separately for report and no-report conditions, it was significant neither in the report condition $(p>.36)$ nor in the no-report condition $(p=.13)$. Note also that the corresponding interaction was not significant in the accuracy data $(F<1)$. (All other effects in the ANOVA on RT data were not significant; $p \mathrm{~s}>$.13.) Although we have no explanation for this unexpected interaction, we believe it is cautious not to put too much weight on the interpretation of this interaction because, to anticipate, this interaction was clearly nonsignificant in Experiment 2.

\section{EXPERIMENT 2}

We attribute the cross-task compatibility effect to a kind of conceptual priming. That is, visual encoding activates a spatial code (e.g., left) that then affects the maintenance of the code for the precued response in the RT task, depending on whether this code is spatially compatible or incompatible. Note, however, that both the target movement in the visual task and the corresponding nonspeeded verbal response (e.g., "left") refer to the same spatial concept. Hence, the crucial code overlap relation underlying the present cross-task compatibility effect could be that between the visual target and the precued response ( $\mathrm{S}-\mathrm{R}$ across tasks), that between the two responses ( $\mathrm{R}-\mathrm{R})$, or both.
The aim of Experiment 2 was to clarify these code overlap relations. To accomplish this, we changed the experimental instructions for the visual-encoding task in Experiment 2. We did not ask participants to report the movement direction of the visual target, as in Experiment 1, but rather had them report the "origin" of the movement. For example, if the target moved to the right, participants had to report "left." In this way, we tested whether the cross-task compatibility effect is based on S-R cross-task overlap or whether it is based on R-R overlap. As in Experiment 1, we defined cross-task compatibility with reference to the movement direction of the visual target. If the cross-task compatibility effect observed in Experiment 1 were due to S-R cross-task overlap, we would expect to replicate it in Experiment 2. If both the stimulus and the response contribute to this crosstask compatibility effect, however, the cross-task compatibility effect should disappear or lie somewhere in between a complete replication of that found in Experiment 1 and a reversal. Finally, if the cross-task compatibility effect is due entirely to R-R overlap, we would expect the direction of the cross-task compatibility effect to be completely reversed in Experiment 2, relative to that in Experiment 1.

\section{Method}

Participants. Eighteen new participants (12 of them female, 6 male), 17-31 years of age, took part for $€ 7.50$ (about \$7.50).

Apparatus, Stimuli, Procedure, and Design. The apparatus, stimuli, procedure, and design were identical to those in Experiment 1 , with the exception of the instruction for the visual-encoding task. Instead of reporting the movement direction, as in Experiment 1, the participants were asked in Experiment 2 to report its spatial origin. We asked, "Where did the stimulus come from: left or right?"

\section{Results and Discussion}

Visual-encoding task. All data analyses proceeded as in Experiment 1. The ANOVA for percentages correct 
(see Table 1, right part) yielded a significant effect of CTI $\left[F(1,17)=11.77, M S_{\mathrm{e}}=0.007581, p<.01\right]$ and a significant interaction of CTI and TGI $\left[F(1,17)=5.76, M S_{\mathrm{e}}=\right.$ $0.007104, p<.05]$. With the short TGI, prolonging the CTI reduced perceptual accuracy by $6.3 \%$ (from $91.8 \%$ to $85.5 \%$ ), whereas this reduction was only $3.6 \%$ with the long TGI $(90.2 \%$ vs. $86.6 \%)$. That is, we replicated the effect of response preparation (i.e., CTI) on accuracy in the visual task. However, the CTI effect was stronger with the short TGI than with the long TGI, suggesting that the detrimental effect of response preparation with a long CTI on perceptual accuracy is enhanced when the go signal for the prepared response is presented immediately following the visual target. That is, when participants are required to respond immediately, the response preparation process interferes with visual encoding even more strongly than when there is some time to process the visual target before initiation of the prepared response (see the General Discussion section).

There was also a nonsignificant trend toward an interaction of CTI with cross-task compatibility $[F(1,17)=4.04$, $\left.M S_{\mathrm{e}}=0.00267, p=.061\right]$. With a short CTI, performance in compatible trials was more accurate than that in incompatible trials (91.3\% vs. $90.6 \%)$, whereas this trend was in the opposite direction for the long CTI ( $84.7 \%$ vs. $87.4 \%)$. Because there was not the slightest indication for this interaction in Experiment $1(F<1)$ and because the trend for an interaction in the present experiment was not significant, we refrain from interpreting the present data trend. All other effects were clearly nonsignificant ( $p s>.27)$.

Reaction time task. We discarded fewer than $1 \%$ of the correct trials as RT outliers. Table 4 shows mean RTs and Table 5 shows percentages correct as functions of CTI, TGI, cross-task compatibility, and report.

First, we will summarize the effects of response preparation on dual-task process interference. The ANOVA yielded a significant effect for report $[F(1,17)=23.31$, $\left.M S_{\mathrm{e}}=95,442, p<.01\right]$, indicating that RT was longer in the report trials than in the no-report trials (912 vs. $737 \mathrm{msec})$. This effect was not significant for percentage correct $(F<1)$. There was also a main effect of TGI for RTs $\left[F(1,17)=138.45, M S_{\mathrm{e}}=45,390, p<.01\right]$, being due to a longer RT with the short TGI than with the long TGI (972 vs. $677 \mathrm{msec}$ ). For the accuracy data, the main effect of TGI was significant $[F(1,17)=11.38$, $\left.M S_{\mathrm{e}}=0.004617, p<.01\right]$, showing that accuracy was higher with the short TGI than with the long TGI $(98.3 \%$ vs. $95.6 \%$ ), which runs counter to the TGI effect in the RT data. In RTs, TGI and report interacted significantly $\left[F(1,17)=44.10, M S_{\mathrm{e}}=24,882, p<.01\right]$, indicating that the TGI effect was larger in report trials $(419 \mathrm{msec})$ than in no-report trials $(172 \mathrm{msec})$. This interaction was not significant in the accuracy data $(p>.23)$.

TGI interacted with CTI $\left[F(1,17)=47.19, M S_{\mathrm{e}}=961\right.$, $p<.01]$, suggesting that the beneficial effect of the long CTI was larger with the short TGI $(33 \mathrm{msec})$ than with the long TGI, where RT was actually even slightly longer (18 $\mathrm{msec}$ ) with the long CTI than with the short TGI. For the accuracy data, there was also a significant main effect of CTI $\left[F(1,17)=18.59, M S_{\mathrm{e}}=0.0009834, p<.01\right]$, qualified by an interaction of CTI and TGI $[F(1,17)=$ 6.88, $\left.M S_{\mathrm{e}}=0.001206, p<.05\right]$. With the short TGI, prolonging the CTI decreased accuracy by $0.5 \%$ (from $98.6 \%$ to $98.1 \%$ ), whereas this decrease was $2.7 \%$ with the long TGI (from $97.0 \%$ to $94.3 \%$ ). (All other $p$ s $>.16$ in the accuracy data.)

We note that the overall pattern of process interference effects was generally similar to that in Experiment 1 . That is, although we did not replicate the significant main effect of CTI for RTs, we did replicate the CTI $\times$ TGI interaction. The three-way interaction of CTI, TGI, and report, which was significant in Experiment 1, just failed to reach significance in Experiment $2[F(1,17)=3.15$, $\left.M S_{\mathrm{e}}=2,601, p=.094\right]$, but the numerical data pattern was similar to that in Experiment 1. Hence, it appears that the change in the instruction did not change the general pattern of dual-task process interference effects.

Of importance, in Experiment 2, we found that RT was $175 \mathrm{msec}$ longer in the report condition than in the noreport condition but that, unlike Experiment 1, this sizable dual-task interference effect was not significantly counteracted by a corresponding decrease in accuracy. This finding suggests that the RT dual-task interference effect with respect to the manipulation of the TGI is only partly due to a trade-off.

Now we turn to the cross-task compatibility effect. We tested whether the change in the instruction would influence, possibly reverse, the cross-task compatibility effect. In fact, the ANOVA yielded a significant main effect of cross-task compatibility $\left[F(1,17)=5.79, M S_{\mathrm{e}}=\right.$ $2,994, p<.05]$, indicating that RT was $16 \mathrm{msec}$ longer

Table 4

Reaction Times (in Milliseconds) in Experiment 2 as a Function of Report, Target-Go Interval (TGI), Cue-Target Interval (CTI), and Cross-Task Compatibility

\begin{tabular}{|c|c|c|c|c|c|c|c|c|}
\hline \multirow[b]{3}{*}{ Condition } & \multicolumn{4}{|c|}{ Report } & \multicolumn{4}{|c|}{ No Report } \\
\hline & \multicolumn{2}{|c|}{ 100-msec TGI } & \multicolumn{2}{|c|}{1,200 -msec TGI } & \multicolumn{2}{|c|}{ 100-msec TGI } & \multicolumn{2}{|c|}{ 1,200-msec TGI } \\
\hline & $M$ & $\overline{S D}$ & $M$ & $S D$ & $M$ & $\overline{S D}$ & $M$ & $S D$ \\
\hline \multicolumn{9}{|l|}{$\mathrm{CTI}=100 \mathrm{msec}$} \\
\hline Incompatible & 1,113 & 265 & 691 & 156 & 840 & 168 & 652 & 155 \\
\hline Compatible & 1,139 & 273 & 695 & 158 & 861 & 168 & 634 & 147 \\
\hline \multicolumn{9}{|l|}{$\mathrm{CTI}=1,200 \mathrm{msec}$} \\
\hline Incompatible & 1,099 & 313 & 705 & 182 & 786 & 190 & 649 & 158 \\
\hline Compatible & 1,136 & 309 & 722 & 184 & 803 & 204 & 668 & 183 \\
\hline
\end{tabular}


Table 5

Percentages Correct in Experiment 2 as a Function of Report, Target-Go Interval (TGI), Cue-Target Interval (CTI), and Cross-Task Compatibility

\begin{tabular}{|c|c|c|c|c|c|c|c|c|}
\hline \multirow[b]{3}{*}{ Condition } & \multicolumn{4}{|c|}{ Report } & \multicolumn{4}{|c|}{ No Report } \\
\hline & \multicolumn{2}{|c|}{ 100-msec TGI } & \multicolumn{2}{|c|}{ 1,200-msec TGI } & \multicolumn{2}{|c|}{ 100-msec TGI } & \multicolumn{2}{|c|}{ 1,200-msec TGI } \\
\hline & $M$ & $S D$ & $M$ & $S D$ & $M$ & $S D$ & $M$ & $S D$ \\
\hline \multicolumn{9}{|l|}{$\mathrm{CTI}=100 \mathrm{msec}$} \\
\hline Incompatible & 98.9 & 2.2 & 97.4 & 4.1 & 99.1 & 2.7 & 95.4 & 5.1 \\
\hline Compatible & 97.3 & 3.8 & 97.9 & 3.2 & 99.1 & 2.7 & 97.2 & 5.7 \\
\hline \multicolumn{9}{|l|}{$\mathrm{CTI}=1,200 \mathrm{msec}$} \\
\hline Incompatible & 99.0 & 1.7 & 95.4 & 3.9 & 97.7 & 3.8 & 94.0 & 7.4 \\
\hline Compatible & 98.0 & 4.2 & 94.8 & 6.9 & 97.7 & 4.8 & 93.1 & 10.8 \\
\hline
\end{tabular}

in compatible trials than in incompatible trials (see Figure 2). Hence, we found that the cross-task compatibility effect in Experiment 2 was reversed in direction but of comparable size, relative to Experiment 1 (i.e., -16 vs. $21 \mathrm{msec})$. An analysis of the error rates indicated that no effect involving the cross-task compatibility variable was significant. Accuracy was slightly but not significantly worse in compatible trials $(96.9 \%)$ than in incompatible trials $(97.1 \%)$, which corresponds to the reversed crosstask compatibility effect for RT.

The reversal of the cross-task compatibility effect suggests that this effect is primarily due to $\mathrm{R}-\mathrm{R}$ overlap across tasks. The three-way interaction of report, CTI, and cross-task compatibility, which was significant in Experiment 1, was not significant $(F<1)$ for either RT or accuracy. There was a nonsignificant trend toward a TGI $\times$ cross-task compatibility interaction $[F(1,17)=$ $\left.3.21, M S_{\mathrm{e}}=2,178, p=.091\right]$, which was due to the fact that the compatibility effect tended to be larger with the short TGI $(-25 \mathrm{msec})$ than with the long TGI $(-6 \mathrm{msec})$. However, this interaction was not significant for accuracy $(p>.32)$. The trend in the RT data appears to be consistent with the idea that temporal overlap of the two critical task processes (i.e., visual encoding and initiating the prepared response), which is present with the short TGI, leads to increased cross-task interactions (see Koch \& Prinz, 2002, for a discussion).

\section{GENERAL DISCUSSION}

\section{Response Preparation and Dual-Task Process Interference}

In both experiments, we found that response preparation based on the precuing interval (i.e., the CTI) affected RT to the go signal. Specifically, in no-report trials response preparation led to faster responses when the TGI was short, but not when it was long. In contrast, in report trials, in which participants had to encode the visual target, we observed that prolonging response preparation time generally had only very small effects. It thus appears that visual encoding "interrupts" or even offsets the response preparation process. This would be consistent with the idea that visual encoding, or perhaps consolidating and maintaining the encoded information, creates a processing bottleneck that impairs (or postpones) other memory- related processes (even though a capacity-sharing account seems to be feasible, too; see Navon \& Miller, 2002, and Tombu \& Jolicœur, 2002, 2003, for recent discussions of capacity-sharing vs. bottleneck accounts). At any rate, the present data suggest a rather limited amount of response preparation when preparation is interrupted by performance of an intervening visual-encoding task.

An alternative way to explain the present data pattern might be to assume that participants adopt a generally more conservative strategy to perform the RT task on report trials, relative to no-report trials. That is, on noreport trials, participants start to prepare the response immediately upon cue presentation, whereas on report trials, they may simply encode the cue but do not start actually preparing the associated response until the go signal. Further research will have to clarify whether the observed dual-task interference effect on response preparation is due to a structural inability to maintain a prepared state when the intervening visual target is processed or whether the interference effect is due to a strategic "reluctance" to prepare a response in one task and process a visual target in another task.

In fact, processing the intervening visual target produced clear dual-task process interference in the RT task. RT to the go signal was substantially longer with the short TGI than with the long TGI, suggesting that encoding a visual stimulus into short-term memory, or maintaining it for later report, postpones response initiation (shortterm consolidation; see Jolicœur \& Dell'Acqua, 1998; Jolicœur et al., 2002; Koch \& Prinz, 2002). However, the TGI effect was not eliminated altogether in the no-report trials. To account for the remaining effect in the no-report trials, we believe that this effect might have been due to an "aging" foreperiod effect, to residual processing of the target even when this was not required, or to both (see Koch, Metin, \& Schuch, 2003, for a discussion in the context of choice RT tasks).

We note, though, that the RT data were associated with speed-accuracy trade-offs. Possibly, relative to short intervals, the number of trials in which the participants anticipated the go signal might have been larger with long intervals, thus leading to faster responses in correct trials but also to increased error rates (see Schubert, 1999). However, in Experiment 2, we observed that RT was substantially longer in the report trials than in the 
no-report trials, suggesting dual-task encoding costs, and that this effect was not compromised by a corresponding decrease in error rates (see also Azuma, Prinz, \& Koch, 2004; Jolicœur \& Dell'Acqua, 1998; Jolicœur et al., 2002; Koch et al., 2003; Koch \& Prinz, 2002).

We also found dual-task effects of response preparation on accuracy in visual encoding. When we increased the response preparation time for the speeded response, we observed in both experiments that perceptual accuracy in the nonspeeded visual encoding task decreased. Previous studies have demonstrated that response selection in a choice RT task can impair subsequent visual encoding (De Jong \& Sweet, 1994; Jolicœur, 1999; Müsseler \& Wühr, 2002). In our experiments, we extended these findings to the effect of preparing a precued response on visual encoding. This suggests that response preparation and visual encoding do not proceed in parallel.

However, an alternative explanation for the decrease of perceptual performance with the long CTI might be that this decrease is due not to increasing response preparation during the CTI but, rather, to an unspecific alerting effect of the cue presentation on subsequent visual encoding. Similarly, participants' attentional states with respect to the visual task might change during the prolonged CTI. Presently, it seems difficult to exclude these alternative accounts for the CTI effect in perceptual accuracy. Possibly, the finding of Experiment 2 that the CTI effect was significantly more pronounced with the short TGI rather suggests common processing resources for response preparation and visual encoding; however, because this effect did not show up in Experiment 1, the present evidence is not completely conclusive.

\section{Code Overlap in Dual Tasks}

In the RT task, we found a cross-task compatibility effect in both experiments. Similar cross-task compatibility effects had already been found with two speeded choice RT tasks (Hommel, 1998; Lien \& Proctor, 2000; Logan \& Gordon, 2001). Our data thus suggest that finding cross-task compatibility effects for RT does not require a dual-task situation in which both responses are speeded. Rather, it appears sufficient that a code relevant to the RT task needs to be formed, even if this code does not refer to a response that is to be performed immediately. This cross-task compatibility effect is most likely due to a priming process that is based on the repeated use of codes representing the same spatial concept (e.g., left). That is, visual encoding activates a spatial code that then primes the compatible code for the precued response in the RT task.

The opposite effect-namely, that speeded response selection or initiation produces cross-task compatibility effects in a logically unrelated visual-encoding task-has also been demonstrated repeatedly in the literature (e.g., Müsseler \& Hommel, 1997; Müsseler \& Wühr, 2002; Stevanovski et al., 2003). In these demonstrations, the identification of a left- or right-pointing arrowhead was impaired if the pointing direction spatially corresponded to another, logically unrelated code formed in the context of a different task, giving rise to "blindness" to compatible stimuli (Müsseler \& Hommel, 1997). In principle, we might have observed a similar effect in the present experiments - that is, planning a precued response might have led to impairment in identifying spatially corresponding stimulus movements in the visual-encoding task. However, we did not find such an effect, either in the present experiments or in previous experiments in which the same paradigm was used (see Koch \& Prinz, 2002).

Several factors might explain the difference between the empirical results found with the present paradigm and those found with paradigms using arrowhead stimuli in the visual task. Perhaps less interesting, there are many procedural differences. For instance, Müsseler and Hommel (1997) and Stevanovski et al. (2003) used an adaptive staircase method to adjust the masking, so that participants always performed at intermediate levels in the visual task, whereas no such method was used in the present experiments. However, a more interesting explanation for this empirical difference might be that arrowheads, as compared with spatial movement stimuli, provide spatial information in a more symbolic format. Possibly, suppression effects such as the "blindness" effect with symbolic spatial information (i.e., arrowheads) are based on more abstract levels of representation (see Stevanovski et al., 2003), whereas code overlap with more "direct" spatial codes rather leads to facilitation. Resolving this possibility appears to be an important issue for further research.

With respect to the present cross-task compatibility effect for RT, with the exception of the unexpected threeway interaction in Experiment 1 that did not replicate in Experiment 2, the cross-task compatibility effect was not significantly affected by response preparation. This result corresponds to the finding that compatibility effects in single-task conditions are known to persist even with prepared responses (e.g., Hommel, 1996; Ivanoff, 2003; Kunde, Koch, \& Hoffmann, 2004). The persistence of the present cross-task compatibility effect is also indicated by the finding that the cross-task compatibility effect was not significantly smaller in no-report trials than in report trials. Converging evidence from a study with a choice RT task, instead of the present precued response task, suggests that this persistence is relatively long-lasting (i.e., it occurs even when report conditions are blocked; see Azuma et al., 2004). However, in contrast to the crosstask compatibility effect, we observed that the dual-task process interference effect was markedly reduced by the intention not to consolidate the visual target in no-report trials. The strong influence of the report manipulation on process interference, as opposed to its small and nonsignificant influence on the cross-task compatibility effect, suggests that the latter is due to an unintentionally activated code, whereas the former is strategically mediated by the intention of the participants to consolidate this code into short-term memory for later report (Jolicœur \& Dell'Acqua, 1998).

Even though the cross-task compatibility effect appears to be unintentional, we found in Experiment 2 that changing the encoding instruction and, thus, the associ- 
ated response in the visual task reversed the direction of the cross-task compatibility effect. Recently, a similar instruction effect on a dual-task code overlap effect has been reported for accuracy effects in a nonspeeded visual identification task (Stevanovski, Oriet, \& Jolicœur, 2002). However, to our knowledge, the present reversal of a cross-task compatibility effect in an RT task is a new phenomenon.

Note that a reversal of a compatibility effect for RT as a function of encoding instruction has been reported several times for S-R compatibility within a task in both singletask contexts (e.g., De Jong, Liang, \& Lauber, 1994) and dual-task contexts (Lien \& Proctor, 2000). For instance, in Lien and Proctor's (2000) dual-task study, a compatibility effect (i.e., the Simon effect) was reversed with a spatially incompatible S-R mapping, but this reversal was confined to code overlap within one and the same task, and the instruction manipulation did not affect cross-task compatibility effects. In contrast, the present reversal refers to the reversal of a code overlap effect across tasks.

Such instruction effects on dual-task cross-task compatibility effects generally suggest the importance of the role of task set for dual-task interactions (e.g., Logan \& Gordon, 2001; Logan \& Schulkind, 2000; Schuch \& Koch, 2004). More specifically, in the present context, the reversal of the cross-task compatibility effect suggests that this effect cannot be based on the physical stimulus, because this was identical in Experiments 1 and 2. Instead, the reversal of the cross-task compatibility effect suggests that it is based on the interpretation of the stimulus. We argue that, in the context of the present task, the code representing this interpretation in terms of the left versus right distinction is identical with the corresponding response code for the deferred verbal report. If that is true, the reversal of the cross-task compatibility effect would indicate that this effect is based on $\mathrm{R}-\mathrm{R}$ overlap, rather than on S-R overlap, across tasks. Importantly, finding evidence suggesting that cross-task compatibility is based on $\mathrm{R}-\mathrm{R}$ overlap seems to imply that the dual-task process interference effect in the RT task may actually be due to the immediate construction of the response code in the context of the visual task. It should be noted, though, that the corresponding response is deferred, unlike standard psychological refractory period experiments with two speeded RT tasks. Yet we find the same kind of dual-task interference effect.

We believe that this is an important finding. If we consider the construction of a cognitive code referring to the identity of a deferred response a response selection, and if this occupies the same cognitive machinery that is also needed to select a response for immediate execution, our results would be consistent with a response selection bottleneck account (Pashler, 1994). We believe, however, that this would broaden the notion of response selection to comprise any cognitive operation that produces a discrete code, thus supporting the proposal of broadening dual-task bottlenecks to comprise interference between memory operations in general (Jolicœur et al., 2002; Pashler, 2000).

\section{Conclusions}

In summary, in a dual-task response-cuing paradigm, we found that encoding a visual target impairs initiation of a precued and prepared response. Also, preparing a precued response impaired accuracy in an unrelated visual-encoding task, but conversely, the process of visual encoding markedly reduced the potential benefit of having prepared the cued response on response initiation. Furthermore, we found a cross-task compatibility effect that reversed its direction when the instruction changed the response associated with the stimulus in the visualencoding task. This suggests that cross-task compatibility is based on $\mathrm{R}-\mathrm{R}$ overlap. Together, these results support the idea that response processes (i.e., preparation and initiation) and visual encoding share, to some degree, the same cognitive codes and processes (e.g., Arnell \& Duncan, 2002; Hommel, Müsseler, Aschersleben, \& Prinz, 2001).

\section{REFERENCES}

Arnell, K. M., \& Duncan, J. (2002). Separate and shared sources of dual-task cost in stimulus identification and response selection. Cognitive Psychology, 44, 105-147.

Azuma, R., Prinz, W., \& Koch, I. (2004). Dual-task slowing and effects of cross-task compatibility. Quarterly Journal of Experimental Psychology, 57A, 693-713.

Carrier, L. M., \& Pashler, H. (1995). Attentional limits in memory retrieval. Journal of Experimental Psychology: Learning, Memory, \& Cognition, 21, 1339-1348.

De Jong, R., Liang, C.-C., \& Lauber, E. (1994). Conditional and unconditional automaticity: A dual-process model of effects of spatial stimulus-response correspondence. Journal of Experimental Psychology: Human Perception \& Performance, 20, 731-750.

De Jong, R., \& SweET, J. B. (1994). Preparatory strategies in overlappingtask performance. Perception \& Psychophysics, 55, 142-151.

Hommel, B. (1996). S-R compatibility effects without response uncertainty. Quarterly Journal of Experimental Psychology, 49A, 546-571.

Hommel, B. (1998). Automatic stimulus-response translation in dualtask performance. Journal of Experimental Psychology: Human Perception \& Performance, 24, 1368-1384.

Hommel, B., Müsseler, J., Aschersleben, G., \& Prinz, W. (2001). The theory of event coding (TEC): A framework for perception and action. Behavioral \& Brain Sciences, 24, 849-937.

Hommel, B., \& PRINZ, W. (EDs.) (1997). Theoretical issues in stimulusresponse compatibility. Amsterdam: Elsevier.

IVANOFF, J. (2003). On spatial response code activation in a Simon task. Acta Psychologica, 112, 157-179.

Jolicceur, P. (1999). Dual-task interference and visual encoding. Journal of Experimental Psychology: Human Perception \& Performance, 25, 596-616.

Jolicceur, P., \& Dell'ACQuA, R. (1998). The demonstration of shortterm consolidation. Cognitive Psychology, 36, 138-202.

Jolicceur, P., \& Dell'ACQUA, R. (1999). Attentional and structural constraints on visual encoding. Psychological Research, 62, 154-164.

Jolicceur, P., Tombu, M., Oriet, C., \& Stevanovski, B. (2002). From perception to action: Making the connection. In W. Prinz \& B. Hommel (Eds.), Common mechanisms in perception and action: Attention and performance XIX (pp. 558-586). Oxford: Oxford University Press.

Koch, I., Metin, B., \& Schuch, S. (2003). The role of temporal uncertainty for process interference and code overlap in perception-action dual tasks. Psychological Research, 67, 244-252.

Koch, I., \& PRINZ, W. (2002). Process interference and code overlap in dual-task performance. Journal of Experimental Psychology: Human Perception \& Performance, 28, 192-201.

Kornblum, S., Hasbroucq, T., \& Osman, A. (1990). Dimensional overlap: Cognitive basis for stimulus-response compatibility. A model and taxonomy. Psychological Review, 97, 253-270. 
Kunde, W., Koch, I., \& Hoffmann, J. (2004). Anticipated action effects affect the selection, initiation, and execution of actions. Quarterly Journal of Experimental Psychology, 57A, 87-106.

Lien, M.-C., \& Proctor, R. W. (2000). Multiple spatial correspondence effects on dual-task performance. Journal of Experimental Psychology: Human Perception \& Performance, 26, 1260-1280.

Lien, M.-C., \& Proctor, R. W. (2002). Stimulus-response compatibility and psychological refractory period effects: Implications for response selection. Psychonomic Bulletin \& Review, 9, 212-238.

Logan, G. D., \& Gordon, R. D. (2001). Executive control of visual attention in dual-task situations. Psychological Review, 108, 393-434.

Logan, G. D., \& Schulkind, M. D. (2000). Parallel memory retrieval in dual-task situations: I. Semantic memory. Journal of Experimental Psychology: Human Perception \& Performance, 26, 1072-1090.

Meyer, D. E., \& Kieras, D. E. (1999). Précis to a practical unified theory of cognition and action: Some lessons from EPIC computational models of human multiple-task performance. In D. Gopher \& A. Koriat (Eds.), Attention and performance XVII: Cognitive regulation of performance. Interaction of theory and application (pp. $17-$ 88). Cambridge, MA: MIT Press.

Müsseler, J., \& Hommel, B. (1997). Blindness to response-compatible stimuli. Journal of Experimental Psychology: Human Perception \& Performance, 23, 861-872.

Müsseler, J., \& WüHR, P. (2002). Response-evoked interference in visual encoding. In W. Prinz \& B. Hommel (Eds.), Common mechanisms in perception and action: Attention and performance XIX (pp. 520-537). Oxford: Oxford University Press.

Navon, D., \& Miller, J. (2002). Queuing or sharing? A critical evaluation of the single-bottleneck notion. Cognitive Psychology, 44, 193-251.

PAshler, H. (1993). Dual-task interference and elementary mental mechanisms. In D. E. Meyer \& S. Kornblum (Eds.), Attention and performance XIV: Synergies in experimental psychology, artificial intelligence, and cognitive neuroscience (pp. 245-264). Cambridge, MA: MIT Press.

PAshler, H. (1994). Dual-task interference in simple tasks: Data and theory. Psychological Bulletin, 116, 220-244.

PAshler, H. (2000). Task switching and multitask performance. In S. Monsell \& J. Driver (Eds.), Control of cognitive processes: Attention and performance XVIII (pp. 277-307). Cambridge, MA: MIT Press.

SCHUBERT, T. (1999). Processing differences between simple and choice reactions affect bottleneck localization in overlapping tasks. Journal of Experimental Psychology: Human Perception \& Performance, 25, 408-425.

ScHUCH, S., \& KocH, I. (2004). The costs of changing the representation of action: Response repetition and response-response compatibility in dual tasks. Journal of Experimental Psychology: Human Perception \& Performance, 30, 566-582.

Stevanovski, B., Oriet, C., \& Joliceeur, P. (2002). Blinded by headlights. Canadian Journal of Experimental Psychology, 56, 65-74.

Stevanovski, B., Oriet, C., \& Jolicceur, P. (2003). Can blindness to response-compatible stimuli be observed in the absence of a response? Journal of Experimental Psychology: Human Perception \& Performance, 29, 431-440.

TOMBu, M., \& Jolicceur, P. (2002). All-or-none bottleneck versus capacity sharing accounts of the psychological refractory period phenomenon. Psychological Research, 66, 274-286.

Tombu, M., \& Joliceur, P. (2003). A central capacity sharing model of dual-task performance. Journal of Experimental Psychology: Human Perception \& Performance, 29, 3-18.

(Manuscript received February 6, 2003; revision accepted for publication October 17, 2004.) 